Background The Pharmacy Department is involved in providing information and recording adverse drug reactions (ADRs) in the national system. The Oncology/Day Hospital provides clinical data. This study increases the culture of safety and security of processing through the collection of data, helping to give statistical and epidemiological value to otherwise casual observations.

Purpose To detect adverse drugs events in oncohaematology in a systematic and timely manner: the FARMAREL project.

Materials and Methods Following the training sessions at the regional level, meetings were held every three months, to monitor progress and analyse any problems found. All haematological patients treated from April 2009 to July 2012 were monitored and if ADRs occurred, a team of physicians and pharmacists analysed the event according to the World Health Organization definition.

The ADRs observed were posted to the network using special software, set up specifically to allow computerization and real-time monitoring progress of the project, as well as statistical analysis of epidemiological data.

Results We reported a total of 74 cases, categorised by the severity of adverse events ( 38 not severe, 3 deaths, 3 life-threatening, 30 hospitalizations or extended hospitalisation). Among the ADRs reported the most significant clinical cases in terms of severity were: Gram-negative septic shock (suspect drug: thalidomide), intestinal infarction (bortezomib), acute renal failure (amphotericin B); hypokinetic cardiomyopathy (doxorubicin); atrioventricular conduction block (lenalidomide). The most significant case studies were presented and discussed with other participating hospitals during a meeting of Lombardy Region, and in a national conference.

Conclusions The study has increased the culture of pharmacovigilance and awareness of the clinical data constituting ADRs. The present evaluation has revealed opportunities for intervention especially for the preventable ADRs which will help in promoting safer drug use.

No conflict of interest.

\section{CPC-075 INTERDISCIPLINARY TASKFORCE BRINGS DOWN PRICE OF HIV DRUGS!}

doi:10.1136/ejhpharm-2013-000276.532

D Tomsen, HB Armandi, A Friberg, H McNulty, LB Skovsted. Region Hovedstadens Apotek, Clinical Pharmaceutical Services, Herlev, Denmark

Background The board in the Danish Regions decided on a new specialist consultancy structure called 'The Council for Use of Expensive Hospital Medicine' (RADS). The aim of RADS is to help standardise the rational use of medicine throughout Denmark, to be achieved primarily by setting guidelines for the use of expensive hospital medicine at the clinical level. The intention is to obtain the best healthcare in relation to expenditure whilst ensuring a high quality of treatment.

Purpose The purpose of this study was to identify an effective way of implementing the RADS guidelines in a multi-centred clinical practise and optimise the pressure on the pricing of the drugs concerned. This was exemplified using data on HIV treatment.

Materials and Methods The task was to change the HIV-treatment from a triple compound to three single compounds. To implement the RADS guidelines, the Capital Regional Pharmacy formed a taskforce consisting of the pharmacy director, top leaders from logistics and clinical pharmaceutical services, IT-department and a data-expert on medication use analysis. The implementation of the HIV-guideline was followed in each clinic during which time the leadership was in close dialogue with the clinicians. Feedback on actual prescribing behaviour was supplied every month to the responsible clinician.

Results The national goal for guidelines implementation was $95 \%$. At $98 \%$ the Capital Region has the highest rate for guidelines implementation in Denmark. Following the next tender and one year after guidelines implementation, the price of the triple compound had dropped by $16 \%$. Result - the price of the clinicians' first choice medicine was acceptable to the Region.

Conclusions The interdisciplinary taskforce achieved its goals. Intensive monitoring and feedback to the clinician in charge, followed by direct management involvement and support at all centres, is an effective implementation strategy.

No conflict of interest.

\section{CPC-076 INTERNAL AUDIT ON THE LABELLING OF INVESTIGATIONAL MEDICINAL PRODUCTS}

doi:10.1136/ejhpharm-2013-000276.533

M Naud, N Gastaut, C Breuker, A Castet-Nicolas, S Hansel-Esteller. CHRU de Montpellier, Pharmacy, Montpellier, France

Background The purpose of labelling is to protect persons who take part to biomedical research. It must enable the product and study to be identified and the drugs to be used safely. The decree of 24 May 2006 [1] sets out the information to be included on the labelling of investigational medical product (IMPs).

Purpose To evaluate the regulatory conformity of the labelling of IMPs.

Materials and Methods An assessment grid was established from the decree of 24 May 2006. This audit investigated the labelling of the primary or secondary packaging, according to the presentation, of 135 IMPs corresponding to 75 clinical trials.

Results Of 135 labels analysed, only 11 (8.1\%) bore all the information required by the legislation. On 3 labels, information didn't appear in French. In more than $5 \%$ of the cases, information allowing identification of the product and the study and the good use of the drugs was absent from label. In other cases the following was missing: pharmaceutical form (15.4\%), route of administration (15.3\%), content of the active substance $(11.6 \%)$, product identification $(6.88 \%)$, clinical trial reference $(6.88 \%)$, patient visit number $(71.9 \%)$ and storage conditions (14.4\%). 57.8\% of the labels came in layers. Basic information was not present on the first layer in $26.1 \%$ of the cases for the pharmaceutical form, route of administration (55.9\%), dosage (13.8\%), product identification (11.7\%) or storage conditions (45.8\%).

Conclusions In spite of important and rigorous regulation, we noted non-conformities in labelling with sometimes important omissions. The significant number of statements required to appear on the label leads sponsors to reduce font size and to present the labels in layers. This audit highlights that the significant amount of information on the label makes it difficult to read and can lead to medicines errors, especially in elderly patients.

\section{Reference}

1. Order of 24 May 2006 establishing the content for the labelling of investigational medicinal products published in France's official journal on 30 May 2006.

No conflict of interest.

\section{CPC-077 INVOLVING PHARMACY TECHNICIANS IN MEDICINES RECONCILIATION IN THE EMERGENCY DEPARTMENT: WHAT CAN WE EXPECT?}

doi:10.1136/ejhpharm-2013-000276.534

'E Cousein, ' $1 \mathrm{M}$ Coussemacq, ${ }^{2} \mathrm{~A}$ Baranyaï, ${ }^{2} \mathrm{P}$ Coupé. 'Valenciennes' Hospital, Pharmacy, Valenciennes, France; 'Valenciennes' Hospital, Emergency, Valenciennes, France

Background In 2011, the Centre Hospitalier de Valenciennes Emergency Department (ED) treated an average of 140 patients per day, and $38.8 \%$ of these patients were hospitalised. Thus, 54 patients a day were eligible for medicines reconciliation at admission.

A previous study showed that the medicines reconciliation of $46.4 \%$ of the patients admitted at the Centre Hospitalier de 
Valenciennes ED was incorrect, and that pharmacists' involvement could improve information gathering about home medicines.

During the study, pharmacists did not find any discrepancies with home meds or any drug-related problems (DRPs) in $38.2 \%$ of the patients. Pharmacists did not add value for these patients.

Separately, the Centre Hospitalier de Valenciennes pharmacy has automated the drug dispensing process. As a result, pharmacy technicians have expressed their reluctance to only work with a machine, fearing they might lose part of their skills in medicines management.

Before this problem arose, it has been proposed that technicians take part in medicines reconciliation in the ED.

Purpose To assess which tasks could be conducted by a pharmacy technician in medicines reconciliation.

Materials and Methods Technicians were present at the ED with a pharmacist. Technicians conducted standardised procedures, such as contacting the community pharmacy or assessing patients' compliance according to scores, and reported the conclusions to the pharmacist.

Results Pharmacy technicians had a strong incentive to get involved, as it refreshed their knowledge of medicines management. Moreover, it helped pharmacist to reconcile more patients in the ED, and to focus on patients with DRPs.

However, pharmacy technicians need to be trained on how to detect DRPs, such as therapeutic escalation, and on how to conduct a patient interview.

Conclusions Involving pharmacy technicians in medicines reconciliation may help the pharmacist in the ED, and allow the technicians to keep up their medicines management skills.

No conflict of interest.

\section{CPC-078 IPILIMUMAB FOR ADVANCED MELANOMA: DRUG USE REVIEW}

doi:10.1136/ejhpharm-2013-000276.535

'AR Rubio Salvador, '2J Medina Martínez, 'JM Martínez Sesmero, 'P Moya Gómez, ${ }^{2}$ MA Cruz Mora, ${ }^{2} \mathrm{JI}$ Chacón López-Muñiz, ' $J J$ Cía Lecumberri. 'Hospital Virgen de la Salud, Pharmacy, Toledo, Spain; ${ }^{2}$ Hospital Virgen de la Salud, Oncology, Toledo, Spain

Background Ipilimumab is a recombinant, fully human monoclonal antibody (IgG1) which blocks the inhibitory effects of cytotoxic T-lymphocyte antigen 4 (CTLA4), a negative regulator of T-cell activation. It has been approved for the treatment of unresectable or metastatic melanoma in patients who have failed or do not tolerate other systemic treatment for advanced disease.

Purpose To review the effectiveness and safety profile of ipilimumab in the treatment of adult patients with advanced melanoma. Materials and Methods Medical record review and retrospective analysis (January 2011 to September 2012) of prescriptions recorded in the Integral Oncology Patient Information System (ONCOBASS) in a teaching general hospital. Previous drug use, dose, line of chemotherapy, number of cycles administered, objective response rate and toxicity were analysed.

Results A total of 5 patients with metastatic melanoma were prescribed ipilimumab (2 male, 3 female), median age 45 (36-60). The 4 cycles of treatment planned were completed by 3 patients, 1 continues in active treatment at the moment of finishing this study and the other one has been lost to follow-up due to change of hospital.

In the group of four patients who received treatment, 2 were prescribed ipilimumab as a second line after failure of a temozolomide-based regimen, and 2 were prescribed ipilimumab as third line after two regimens based on immunotherapy, temozolomide or vemurafenib.

After completing the 4 cycles planned, 1 patient maintained complete response (16 months) and 1 patient showed stable disease (maintained for 5 months), and the other one is in evaluation.

No patients suffered grade 3-4 toxicity and the treatment was well tolerated.
Conclusions Ipilimumab has shown effectiveness and safety in the treatment of unresectable or metastatic melanoma in patients who have failed or do not tolerate other systemic treatment for advanced disease in our patients, although data from more patients and longer-term studies are required.

No conflict of interest.

\section{CPC-079 MANAGEMENT OF MYELODISPLASTIC SYNDROMES AND LYMPHOMAS: THE EXAMPLE OF LENALIDOMIDE}

doi:10.1136/ejhpharm-2013-000276.536

${ }^{1} \mathrm{M}$ Scaldaferri, ${ }^{1} \mathrm{E}$ Sciorsci, ${ }^{1} \mathrm{~F}$ Re, ${ }^{2} \mathrm{C}$ Calvo, ${ }^{3} \mathrm{M}$ Chiumente, ${ }^{3} \mathrm{D}$ Barilà, ${ }^{3} \mathrm{~A}$ Chiesa, ${ }^{3}$ M Ferroni, 'S Stecca, 'F Cattel. 'A. O.U. San Giovanni Battista, Pharmacy, Turin, Italy; ${ }^{2}$ University of Turin, Faculty of Pharmacy, Turin, Italy; ${ }^{3}$ University of Turin, School of Hospital Pharmacy, Turin, Italy

Background At our centre, haematologists and department pharmacists constantly monitor outcomes and safety of treatment with lenalidomide.

Purpose To describe clinical outcomes and safety of lenalidomide in our lymphoma and myelodysplastic syndrome patients.

Materials and Methods Onco-AIFA Registry and medical records were checked as of 30/06/2012 for diagnosis, duration of treatment, incidence of adverse drug events (ADRs).

Results Data of 34 patients were reviewed, with the following diagnoses: Diffuse large B-cell lymphoma (DLBCL), 24 patients; $5 \mathrm{q}$-myelodysplastic syndrome (MDS5q-), 11 patients and mantle cell lymphoma (MCL), one patient.

Of patients with DLBCL, one discontinued treatment because of serious ADRs, two because of death and 4 for disease progression after an average of 4.4 treatment cycles, corresponding to 7 months (range: $2-18$ ).

Of patients with MDS5q-, 8 stopped treatment, two of whom because of disease progression or death and two for toxicity. The median duration of treatment was 11.8 cycles (range 1-29).

Seventeen DLBCL patients and 3 MDS5q- patients are still on therapy.

34 non-serious $\mathrm{ADR}$ s relating to 14 patients and 5 serious $\mathrm{ADR}$ relating to 4 patients were reported, two of which were cases of development of solid neoplasia. Non-serious ADRs were mostly cases of haematological toxicity, alterations of the skin and of nervous system and infections.

Conclusions Lenalidomide seems to control the disease in patients with MDS5q- for long periods, while the Time to Progression in patients with DLBCL appears shorter.

The treatment-related toxicity appears in most cases acceptable.

Despite the limited number of data, our analysis highlights the need for close monitoring of the patients both during treatment and on follow-up, as evidenced by the two cases of onset of neoplasia.

The progressive collection of data is providing the haematologists and pharmacists the information to design a model for optimised appropriate treatment with lenalidomide.

No conflict of interest.

\section{CPC-080 MANAGEMENT OF POSTOPERATIVE PAIN AT MOHAMMED V MILITARY TEACHING HOSPITAL, RABAT, MOROCCO}

doi:10.1136/ejhpharm-2013-000276.537

'W Enneffah, "I Zakariya, 'MA El Wartiti, ${ }^{2} \|$ Fabrice, ${ }^{3} \mathrm{~N}$ Cherkaoui, ${ }^{1} \mathrm{~A}$ Bennana. ${ }^{1}$ Mohammed V Military Teaching Hospital - Faculty of Medicine and Pharmacy, Clinical Pharmacy - Therapeutic Chemistry, Rabat, Morocco; ${ }^{2}$ Faculty of Medicine and Pharmacy - Mohammed V University, Souissi, Rabat, Morocco; ' 3 ohammed V Military Teaching Hospital - Faculty of Medicine and Pharmacy, Pharmacy - Galenic Pharmacy, Rabat, Morocco 